\title{
Application of a Novel Tongue Resin Replication System for in vitro Biofilm Studies
}

\author{
Sara Bernardi ${ }^{*}$, Martin Grootveld ${ }^{2}$, Jyoti Tejpal ${ }^{2}$ and Katie Laird ${ }^{2}$ \\ ${ }^{1}$ Department of Life, Health and Environmental Sciences, University of L'Aquila, Italy. \\ ${ }^{2}$ School of Pharmacy, De Montfort University, Leicester, LE1 9BH, UK.
}

\section{Authors' contributions}

This work was carried out in collaboration between all authors. Author SB designed and developed the impressed tongue resin device, performed the laboratory microorganism culture experiments, and also contributed to the writing and editing of the manuscript. Author MG designed the experiments, performed statistical analysis of the experimental dataset, and also contributed to the writing and editing of the manuscript. Author JT performed the microorganism culture count experiments and author KL designed the experiments and contributed to the writing and editing of the manuscript. All authors read and approved the final manuscript.

Article Information

DOI: $10.9734 / B M R J / 2016 / 28606$

Editor(s):

(1) Kai Zhang, Department of Oral Biology, The State University of New York, Buffalo, USA.

Reviewers:

(1) Tomasz Jarzembowski, Medical University of Gdańsk, Poland.

(2) Chen-Chin Chang, University of Kang Ning, Taiwan. Complete Peer review History: http://www.sciencedomain.org/review-history/16101

Short Research Article

Received $28^{\text {th }}$ July 2016

Accepted $1^{\text {st }}$ September 2016

Published $8^{\text {th }}$ September 2016

\section{ABSTRACT}

Aims: Biofilms present a strategy for microorganisms to survive and resist adverse conditions. In order to further our understanding of this resistance mechanism, it is of much importance to develop new methodologies in order to assess such biofilm activities. One of the most defined biofilms is dental plaque. However, the oral cavity contains a different class of biofilm, the precise structural nature of which is dependent on its site of development. Indeed, tongue-coated biofilms have remained a focus for in vitro studies, especially since they represent the major source of oral malodor. The aim of this research was to investigate the growth of a biofilm on a tongue replication constructed from a resin material, and its use in further experimental investigations.

Methods: The model used for this study involved an adapted CDC biofilm reactor in order to achieve this; Staphylococcus aureus was used as the test organism.

Results: Biofilm culturing techniques employed demonstrated a positive growth from the microbes 
retrieved from the tongue-impressed resin material. Moreover, results acquired confirmed that the resin-based tongue replication harbored significantly greater levels of $S$. aureus than those of unimpressed resin, plastic and stainless steel controls.

Conclusions: Therefore, this study provides evidence that the employment of a CDC biofilm reactor in combination with a resin tongue device creates an oral biofilm model which provides valuable information regarding our understanding of how the roughness of the dorsal lingual surface affects microbial populations in patients suffering from oral malodor.

Keywords: Biofilm; tongue coating; CDC biofilm reactor; impression material; halitosis.

\section{INTRODUCTION}

A biofilm is represented by a collection of microbial communities enclosed by a matrix of extracellular polymeric substances (EPSs), and separated by a network of open water channels [1]. The microbes of a biofilm are protected against environmental factors such as UV light and dehydration, in addition to host immune cells such as neutrophils and other phagocytes, together with microbicidal molecules, particularly lactams [2]. In particular, resistance to polymorphic neutrophils has been found in quorum-sensing induced $P$. aeruginosa, where the production of rhamnolipid B, a PMN toxin, was shown to effectively kill the phagocytic cells. Moreover, biofilm-incorporated bacteria exhibit a different phenotype from suspended bacterial cells of the same genotype [3]. Biofilms can develop on both abiotic and biotic surfaces, and act as a source of various infections, such as infective endocarditis and cystic fibrosis pneumonia $[4,5]$.

The area of biofilm-mediated infections can include temporary or permanent medical devices such as endotracheal tubes, intravascular and urinary catheters, orthopedic implants and arterial stents, in addition to wounds, blood, and mucosal surfaces (sinuses, respiratory and genito-urinary tracts) [6-8].

One of the most studied types of biofilms are those formed within the oral cavity, in view of their interactions with biotic and abiotic surfaces that may constitute a form of chronic infection. The most common and documented oral biofilm is dental plaque, responsible for dental caries (aerobic microorganisms such as Streptococcus mutans), and periodontal diseases (anaerobic microorganisms such as Prophyromonas ginggivalis, Actinobacillus actinomycetiemcomitans and Treponema denticola).

A further oral biofilm that is still not well understood is that which coats on the tongue, and which is associated with oral malodour. Indeed, firstly Yaegaki et al. [8] and subsequently Loeshe and Kazor [9], found that the dorsum of the tongue serves as the primary source of volatile sulphur compounds (VSCs). Furthermore, numerous studies have revealed positive correlations between the tongue coating status (presence and/or amount) and a range of differing parameters directly related to oral malodor, and a significant positive correlation was found between oral cavity air levels of VSCs, organoleptic scores of full mouth odor, and the presence or amount of tongue coating observed $[9,10]$. Moreover, de Boever [10] reported a linear Pearson correlation coefficient of 0.63 between total mouth odor and tongue coating level. Furthermore, patients presenting with a significant tongue-coating harbored more bacteria than those without such tongue-coatings $\left[11.9 \times 10^{8}\right.$ vs. $3.8 \times 10^{6}$ Colony Forming Units (CFUs) per sample) $[11,12]$.

Additionally, some researchers have described a relationship between the level of tongue-coating (semi-quantitative scale) and the degree of tongue odour assessed organoleptically $[11,13]$. Firstly, Quirynen et al. [11] hypnothesized that in the absence of a significant correlation between tongue coating and its microbial load, the tongue coating per se, may be responsible for oral malodor. Secondly, Mantilla et al. [13] found that the extent of a white coating on the dorsum of the tongue in periodontitis patients is greater than that observed in healthy volunteers, but they failed to detect a relationship between the presence of such a coating and salivary bacterial load [13].

These possible relationships between tonguecoatings and bacterial load were supported by the detection of benzoyl-arginine naphthylamidehydrolysing bacteria on the tongue in halitosis patients [11]. However, a further report found no association between these coatings and salivary bacterial load in healthy, gingivitis and periodontitis patients (analyzed by means of phase-contrast microscopy) [13]. However, the 
difficulties of studying morphological characteristics of tongue-coating biofilms are strikingly evident in the sampling process and the reproducibility of datasets acquired in vitro.

The aim of this research investigation was to assess the growth of a biofilm on a tongue coating replication synthesized in resin, together with its potential employment in further experimental investigations focused on this research area.

\section{METHODOLOGY}

In order to reproduce an in vitro biofilm, a CDC Biofilm Reactor (CBR 90, Biosurface Technologies Corporation) was utilized. This methodology was based on a continuous flow model in which growth media flowed at a specified rate, and was baffled stirred in order to generate shear force. Biofilm accumulation was then quantified by harvesting the biofilm from coupons of a known surface area, disaggregating the cells and polymeric matrix, and subsequently performing viable plate counts [14-15]. In this experimental design, this procedure was repeated with the coupon system replaced with a tongue model previously cast in resin from a tongue impression taken from a patient suffering from halitosis (Fig. 1). The patient providing the tongue impression had been enrolled voluntarily in the context of a halitosis study currently ongoing at the dental clinic in the University of L'Aquila, and approved by the University of L'Aquila Research Ethics Committee. Six replicate measurements $(n=6)$ were made for all materials tested.

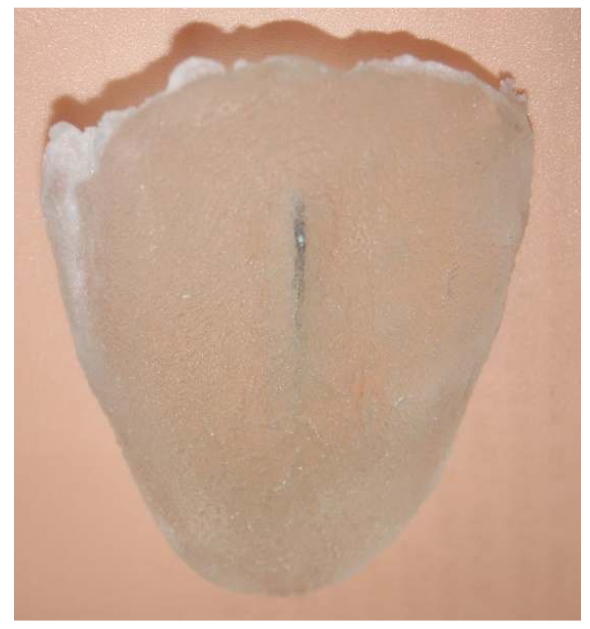

Fig. 1. Representation of resin tongue impression

\subsection{Microorganism and Culture Methods}

All media was obtained from Oxoid Ltd. (Basingstoke, Hampshire, UK) unless otherwise stated. The test organism was Staphylococcus aureus (ATCC 6538). Strains were stored on beads (microbank PL.160/M, Prolab Diagnostics, Cheshire, UK) at $-80^{\circ} \mathrm{C}$. S. aureus was grown on Nutrient Agar (NA), and one colony per $10.0 \mathrm{ml}$ volume of Nutrient Broth (NB) was grown aerobically for $24 \mathrm{hr}$. at $37^{\circ} \mathrm{C}$; this culture was used to inoculate the biofilm reactor. Initial inoculi were computed via spiral plating onto NA.

\subsection{Biofilm Formation}

\subsubsection{Batch phase}

The test materials, i.e. the resin tongue, together with appropriate controls (specifically the unimpressed resin tongue material, together with both plastic and stainless steel coupons) were placed in the biofilm reactor containing $500.0 \mathrm{ml}$ and $1.00 \mathrm{ml}$ volumes of $\mathrm{NB}$ and an overnight culture of $S$. aureus respectively. The reactor was then placed on a stirring plate $(125 \mathrm{rpm})$ and incubated at room temperature for a period of 24 hr (Fig. 2). Authors underline although the tongue is internal and there may be an argument of increased temperature incubation, the ambient temperature was chosen for these studies since elevations in temperature above this level would affect growth rate, and hence also flow-rate and the overall set-up.

\subsubsection{Continuous flow}

Subsequent to a $24 \mathrm{hr}$. period in the batch phase, a continuous flow of media (NB) was passed through the biofilm reactor at a flow-rate of $15.24 \mathrm{ml} / \mathrm{min}$. for $24 \mathrm{hr}$. at room temperature. The flow-rate is defined as the CDC biofilm reactor volume $(350 \mathrm{ml})$ divided by residence time of the bacteria, in order to ensure that $S$. aureus numbers remain static. The doubling time of $S$. aureus was then calculated, resulting in a required residence time of $30 \mathrm{~min}$.

\subsubsection{Enumeration}

The tongue-impressed resin and the above control materials were removed from the biofilm reactor, washed with phosphate-buffered saline (PBS) solution in order to remove planktonic cells, submerged in PBS, and then sonicated for $10 \mathrm{~min}$. at $60 \mathrm{~Hz}$ and 100 Watts. Subsequently, the PBS solution arising therefrom was spiral- 
plated onto nutrient agar and incubated at $37^{\circ} \mathrm{C}$ for $24 \mathrm{hr}$.; colonies were then enumerated. As noted above, relevant control materials employed for this study were resin without a tongue impression, and also plastic and stainless steel coupons.

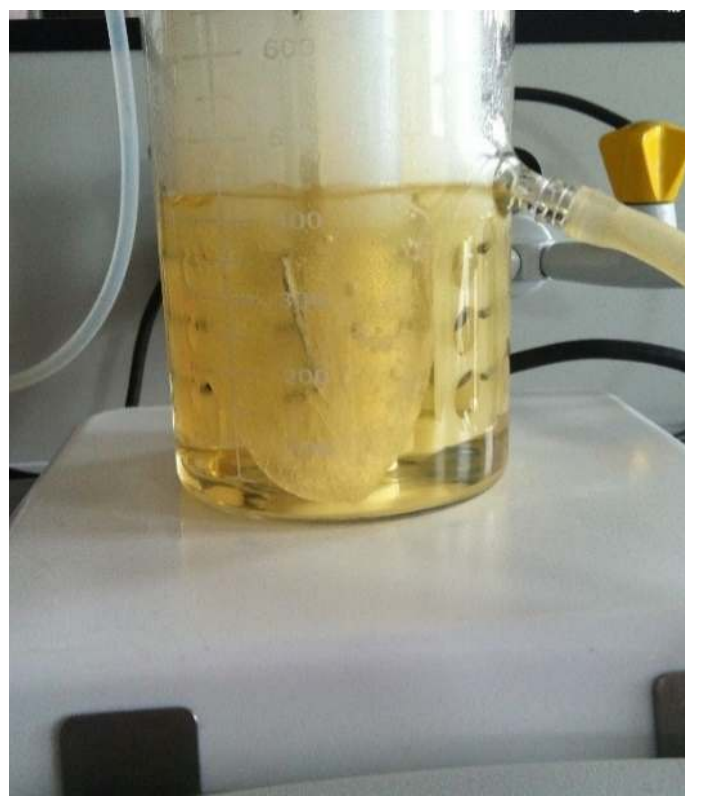

Fig. 2. Pictorial representation of the casted tongue evaluated in a biofilm reactor system

The probe was cleansed by rinsing for $30 \mathrm{~s}$. at $20,500 \mathrm{rpm}$ in sterile water, followed by a rinse at an equivalent rpm value for $15 \mathrm{~s}$ in $70 \%(\mathrm{v} / \mathrm{v})$ ethanol, and then immersed in ethanol for 1.00 min., and finally rinsed with sterile water (again at $20,500 \mathrm{rpm}$ for $30 \mathrm{~s}$ ).

\subsection{Statistical Analysis}

The obtained data counts were statistically analyzed by ANOVA, followed by Tukey's Honestly Significant Difference (HSD) test for individual post-hoc comparisons of the four group mean counts. The primary ANOVA $p$ value was computed using the Welch test statistic, which allows for any inequalities in 'within-sample' variances for each of the four groups compared. A mathematical model for this experimental design is provided in equation 1 , in which $Y_{i}$ represents the (univariate output) $\log _{10} \mathrm{CFU} / \mathrm{ml}$ values observed, $\mu$ their overall population mean value in the absence of any significant, influential sources of variation, $M_{i}$ the 'between-materials' source of variation, and $e_{i}$ the unexplained error (residual) contribution. These analyses were performed with XLSTAT2016 software modules.

$$
\mathrm{Y}_{i}=\mu+\mathrm{M}_{i}+\mathrm{e}_{i}
$$

\section{RESULTS AND DISCUSSION}

The biofilm culturing techniques employed in this investigation showed a positive growth of $S$. aureus biofilm on the resin tongue evaluated, with a $2.91 \log _{10}$ determination of biofilm released from this material, compared with corresponding $8.9 \log _{10}, 5.74 \log _{10}$ and 5.52 $\log _{10}$ values the smooth unimpressed resin surface, plastic and stainless steel control coupons respectively (Fig. 3). This difference is ascribable to significant obstructions to the retrieval of the biofilm presented by the rough surface of the impressed tongue resin material when expressed relative to that of a corresponding smooth (control) one, and, for the non-resin coupons, also to the differing types of materials used as substrates. The HSD test revealed that all post-hoc comparisons were significant (i.e. $p<10^{-9}$ ), with the exception of the SS Control vs. Plastic Control comparison.

Since biofilms represent the most common "lifestyle" of microbes, investigations of their mechanisms of growth on different surface environments involved in human diseases are of paramount importance and hence may enable new antimicrobial agents to be developed in order to eradicate them from pathological processes. Traditionally, oral plaque has always been studied in vitro or ex-vivo on similar enamel surfaces [16]. The tongue biofilm, however, has always been characterized in vitro by molecular and culturing techniques [17,18], and the models used to explore it have never considered the irregular surface on which this biofilm has the capacity to grow and proliferate (this may be ascribable to the difficulties involved in sampling processes). Importantly, the proposed model demonstrated here can be employed in ex-vivo studies, which successfully reproduce an accurate template for the oral environment.

In addition to infectious diseases, interests in biofilm formation has grown within the food industry, in particular approaches for the assessment of biofilm formation in food and water contaminants, together with metal surface corrosion and clogging. Biofilm formation has been demonstrated for numerous pathogens, and is clearly one of the main strategies for bacterial survival in a variety of sites within the human body, and also on inanimate surfaces [1]. In order to enhance our knowledge and to understand biofilm physiology, biofilm model systems are required to be developed in order to 
assess these complex communities under controlled conditions [19-21].

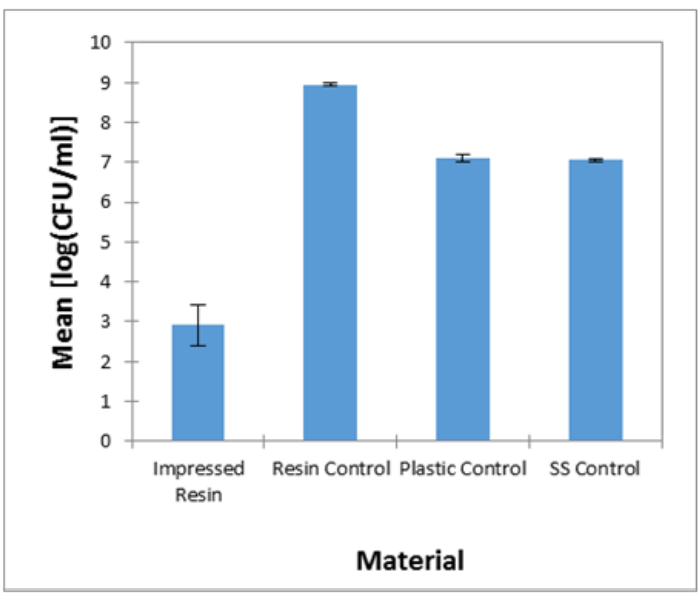

Fig. 3. Bar diagram showing plots of mean released S. aureus counts $\left[\log _{10}(\mathrm{CFU} / \mathrm{ml})\right]$ with associated $95 \%$ confidence intervals for the four material groups tested Abbreviations: SS, Stainless Steel

In view of the multiplicity/heterogeneity of oral biofilm populations, simultaneous multispecies evaluations of the oral microcosm are of a high level of complexity [22,23]. However, the utilisation of an in vitro model system with a highly complex bacterial diversity, and which also supports the growth of uncultivated oral species, is highly desirable, since it may be manipulated and explored within a controlled environment [23].

In the CDC biofilm reactor, the magnetic stirrer within the centre of the vessel provides the shear force and continuous mixing of the device's bulk fluid (introduced in the reactor by means of a peristaltic pump) over the colonised surfaces [24]. Indeed, statistical analysis performed by Goeres [25] demonstrated that the CDC biofilm reactor system serves as a reliable experimental tool to study biofilm formation evolving from a wide range of organisms.

In the reactor system, 24 identical biofilms may be formed simultaneously, and in view of the nature of the reactor components, it permits the facile removal of discs during the experimental procedure, an advantage which customizes the system to the time-dependent study of biofilm formation $[26,27]$. The CDC biofilm reactor model used in the study conducted by Rudney et al. [21] has been shown to be able to produce reproducible microcosm biofilms that were representative of the oral microbiota. Notably, they were able to produce biofilm growth on disks of hydroxyapatite (HA), silorane-based substrates (LS), together with methacrylatebased composites (Z100), which were $12 \mathrm{~mm}$ in diameter. Basal mucin medium (BMM) was used as the growth medium, whilst saliva and dental plaque samples were utilised as inoculi. Both of the two substrates showed a positive biofilm growth, but with differing microbiota compositions, and which was dependent on the differing natures of the two inoculum sources; salivary flora are more similar to that of the tongue than they are to that of dental plaque [22]. In the model used in this study, the system was adapted to accommodate a resin tongue surface replacing the commonly-employed coupon discs (Fig. 2), so that instead of 24 biofilms being produced, it was only possible to form a single one, albeit one with a larger surface area.

The tongue, with its large surface area, represents a unique ecological niche within the oral cavity. This promotes the retention of greater amounts of microorganisms thereon, and therefore it can harbor an extensive bacterial population. Notwithstanding, relatively little is known about the organization of the tongue flora, although it is generally accepted that they form biofilm microenvironments. The bacterial composition of the oral cavity has been shown to include Streptococci sp. and Actinomyces sp., Fusobaterium, Porphyromonas and Enterobacteria [28-30].

However, correlations between the roughness of the dorsal lingual surface, tongue-coated biofilms and oral malodour biomarkers (particularly VSCs in exhaled human breath) remain to be elucidated. In view of their inner nature, the presence of fissures and grooves constitutes a reservoir for harboring microbiota, and hence provides an ecological niche for them. Interestingly, previously published research work has related the presence of deep fissures to higher total counts of bacteria (approximately a 2 -fold change), and also to significantly higher mouth and tongue odour scores [10], although further investigations have failed to confirm any associations of elevated bacterial counts with an increased level of tongue surface roughness $[11,13]$. Similar results were obtained in this study, with microbial counts released from the control resin being much higher than that from the resin tongue impression. In order to further address this issue, many clinical trials, i.e. those 
involving both in vivo and in vitro models, have been postulated and explored [31-34]. However, in view of the inherent features of the tongue and biofilms thereon, the precise nature of relationships between surface roughness and the nature of such biofilms remains questionable. Notwithstanding, the results reported here demonstrate the efficacy and possible future employment of a CDC biofilm reactor, i.e. one which successfully replicates the tongue dorsal surface, for the development of a new model for assessing the oral microcosm within tongue coatings. It is therefore anticipated that this study will stimulate further investigations in this research area.

\section{CONCLUSION}

Although only pilot data was collected in this study, these experiments demonstrated the possibility of employing a CDC biofilm reactor in combination with a human-impressed resin tongue device in order to create an oral biofilm model. Indeed, the results acquired clearly demonstrated that the rough surface of this material presented a significant barrier to the release of $S$. aureus biofilm. This experimental approach may enhance our understanding of the mechanisms involved in the ability of the dorsal lingual surface to affect the microbial population in patients suffering from oral malodor.

\section{ETHICAL APPROVAL}

All authors hereby declare that all experiments have been examined and approved by the appropriate ethics committee and have therefore been performed in accordance with the ethical standards laid down in the 1964 Declaration of Helsinki.

\section{COMPETING INTERESTS}

Authors have declared that no competing interests exist.

\section{REFERENCES}

1. Hall-Stoodley L, Costerton JW, Stoodley P. Bacterial biofilms: From the natural environment to infectious diseases. Nat Rev Microbiol. 2004;2:95-108.

DOI: 10.1038/nrmicro821

2. Dufour $D$, Leung $V$, Lévesque $C M$. Bacterial biofilm: Structure, function, and antimicrobial resistance. Endod Top. 2010; 22:2-16.

DOI: 10.1111/j.1601-1546.2012.00277.x

3. Hall-Stoodley L, Stoodley P. Evolving concepts in biofilm infections. Cell Microbiol. 2009;11:1034-43.

DOI: 10.1111/j.1462-5822.2009.01323.x

4. Ramirez-Ronda $\mathrm{CH}$. Adherence of glucanpositive and glucan-negative streptococcal strains to normal and damaged heart valves. J Clin Invest. 1978;62:805-14.

DOI: $10.1172 / \mathrm{JCl} 109192$

5. Wolcott RD, Dowd SE. A rapid molecular method for characterising bacterial bioburden in chronic wounds. 2008;17.

6. Donlan RM. Biofilm formation: A clinically relevant microbiological process. Clin Infect Dis. 2001;33:1387-92.

DOI: $10.1086 / 322972$

7. Bakaletz LO. Bacterial biofilms in otitis media: Evidence and relevance. Pediatr Infect Dis J 2007;26:S17-9.

DOI: 10.1097/INF.0b013e318154b273

8. Yaegaki K, Sanada K. Biochemical and clinical factors influencing oral malodor in periodontal patients. J Periodontol. 1992; 63:783-9.

DOI: 10.1902/jop.1992.63.9.783

9. Loesche WJ, Kazor C. Microbiology and treatment of halitosis. Periodontol 2000. 2002;28:256-79.

DOI: $10.1007 / \mathrm{s} 11908-003-0077-8$

10. De Boever EH, Loesche WJ. Assessing the contribution of anaerobic microflora of the tongue to oral malodor. J Am Dent Assoc. 1995;126:1384-93.

DOI: 10.14219/jada.archive.1995.0049

11. Quirynen $M$, Mongardini $C$, van Steenberghe $D$. The effect of a 1-stage full-mouth disinfection on oral malodor and microbial colonization of the tongue in periodontitis. A pilot study. 1998;69.

DOI: 10.1902/jop.1998.69.3.374

12. Bosy A, Kulkarni GV, Rosenberg M, McCulloch CA. Relationship of oral malodor to periodontitis: Evidence of independence in discrete subpopulations. J Periodontol 1994;65:37-46.

DOI: 10.1902/jop.1994.65.1.37

13. Mantilla Gómez S, Danser MM, Sipos PM, Rowshani B, van der Velden $U$, van der Weijden GA. Tongue coating and salivary bacterial counts in healthy/gingivitis subjects and periodontitis patients. J Clin Periodontol. $2001 ; 28: 970-8$.

DOI: 281011 [pii] 
14. Williams DL, Bloebaum RD. Observing the biofilm matrix of Staphylococcus epidermidis ATCC 35984 grown using the CDC biofilm reactor. Microsc Microanal. 2010;16(2):143-52.

DOI: $10.1017 / S 143192760999136 \mathrm{X}$

15. Honraet K, Goetghebeur E, Nelis HJ. Comparison of three assays for the quantification of Candida biomass in suspension and CDC reactor grown biofilms. J Microbiol Methods. 2005; 63(3):287-95.

DOI: 10.1016/j.mimet.2005.03.014

16. Zahradnik RT, Propas D, Moreno EC. In vitro enamel demineralization by Streptococcus mutans in the Presence of Salivary Pellicles. J Dent Res. 1977;56: 1107-10.

DOI: $10.1177 / 00220345770560091601$

17. Paster BJ, Olsen I, Aas JA, Dewhirst FE. The breadth of bacterial diversity in the human periodontal pocket and other oral sites. Periodontol 2000. 2006;42:80-7.

DOI: 10.1111/j.1600-0757.2006.00174.x

18. Mashima I, Kamaguchi A, Nakazawa F. The distribution and frequency of oral Veillonella spp. in the Tongue biofilm of healthy young adults. Curr Microbiol. 2011;63:403-7.

DOI: 10.1007/s00284-011-9993-2

19. Pierce JT. Manual of Environmental Microbiology, $3^{\text {rd }}$ edition. American Society for Microbiology Press. 2008;29.

DOI:10.1097/01.shk.0000286267.62940.8 4

20. Hamilton $M$, Heersink J, BuckinghamMeyer K, Goeres D. The biofilm laboratory: Step by step protocols for experimental design, analysis and data interpretation. Cytergy; 2003.

21. Smith DS, Ferris FG, Corbin J, Hardman J. Methods in enzymology volume 336: Microbial growth in Biofilms part A Developmental and Molecular Biological Aspects. 2001;3.

22. Li Y, Carrera C, Chen R, Li J, Lenton P, Rudney JD, et al. Degradation in the dentin-composite interface subjected to multi-species biofilm challenges. Acta Biomater. 2014;10:375-83.

DOI: 10.1016/j.actbio.2013.08.034

23. Rudney JD, Chen R, Lenton P, Li J, Li Y, Jones RS, et al. A reproducible oral microcosm biofilm model for testing dental materials. J Appl Microbiol. 2012;113: 1540-53.

DOI: 10.1111/j.1365-2672.2012.05439.x

24. Coenye $\mathrm{T}$, Nelis HJ. In vitro and in vivo model systems to study microbial biofilm formation. J Microbiol Methods. 2010;83: 89-105.

DOI: 10.1016/j.mimet.2010.08.018

25. Goeres DM, Loetterle LR, Hamilton MA, Murga R, Kirby DW, Donlan RM. Statistical assessment of a laboratory method for growing biofilms. Microbiology. 2005;151: 757-62.

DOI: $10.1099 /$ mic.0.27709-0

26. Honraet K, Goetghebeur E, Nelis HJ. Comparison of three assays for the quantification of Candida biomass in suspension and CDC reactor grown biofilms. J Microbiol Methods. 2005;63: 287-95.

DOI: 10.1016/j.mimet.2005.03.014

27. Nailis $H$, Vandenbroucke R, Tilleman $K$, Deforce $D$, Nelis $H$, Coenye T. Monitoring ALS1 and ALS3 gene expression during in vitro Candida albicans biofilm formation under continuous flow conditions. Mycopathologia. 2009;167:9-17.

DOI: $10.1007 /$ s11046-008-9148-6

28. Goldberg S, Cardash H, Browning $H$, Sahly $H$, Rosenberg $M$. Isolation of Enterobacteriaceae from the mouth and potential association with malodor. J Dent Res 1997;76:1770-5.

DOI: $10.1177 / 00220345970760110801$

29. Gordon DF, Gibbons RJ. Studies of the predominant cultivable micro-organisms from the human tongue. Arch Oral Biol. 1966;11:627-32.

DOI: 10.1016/0003-9969(66)90229-9

30. Hartley MG, El-Maaytah MA, McKenzie C, Greenman J. The tongue microbiota of low odour and malodorous individuals. Microb Ecol Health Dis. 1996;9:215-23.

DOI: 10.3109/08910609609166462

31. Bernardi S, Marzo G, Continenza MA. Dorsal lingual surface and halitosis: $A$ morphological point of view acta stomatol. Croat. 2016;50(2):151-157.

32. Saad S, Hewett K, Greenman J. Use of an in vitro flat-bed biofilm model to measure biologically active anti-odour compounds. Appl Microbiol Biotechnol. 2013;97:786575.

DOI: 10.1007/s00253-013-5084-6 
33. Böl M, Ehret AE, Bolea Albero A, Hellriegel $\mathrm{J}$, Krull R. Recent advances in mechanical characterisation of biofilm and their significance for material modelling. Crit Rev Biotechnol. 2012;1-27.

DOI: 10.3109/07388551.2012.679250
34. Taylor BJ, Greenman J, Salisbury V, Nelson SM. Modelling the effects of $\mathrm{pH}$ on tongue biofilm using sorbarod biofilm perfusion system. J Breath Res. 2009;4: 017107.

DOI: $10.1088 / 1752-7155 / 4 / 1 / 017107$

(c) 2016 Bernardi et al.; This is an Open Access article distributed under the terms of the Creative Commons Attribution License (http://creativecommons.org/licenses/by/4.0), which permits unrestricted use, distribution, and reproduction in any medium, provided the original work is properly cited.

Peer-review history:

The peer review history for this paper can be accessed here: http://sciencedomain.org/review-history/16101 